\title{
Study of gestational trophoblastic diseases at a tertiary care hospital in India
}

\section{Ramalingappa C. Antaratani, Shruthi M.*}

Department of Obstetrics and Gynecology, KIMS, Hubli, Karnataka, India

Received: 05 February 2018

Accepted: 07 March 2018

\section{*Correspondence:}

Dr. Shruthi M.,

E-mail: mshruthi597@gmail.com

Copyright: (C) the author(s), publisher and licensee Medip Academy. This is an open-access article distributed under the terms of the Creative Commons Attribution Non-Commercial License, which permits unrestricted non-commercial use, distribution, and reproduction in any medium, provided the original work is properly cited.

\begin{abstract}
Background: Gestational trophoblastic disease refers to the heterogeneous group of interrelated lesions that arises from abnormal proliferation of placental trophoblasts. GTNs are among the rare human tumours that can be cured even in the presence of widespread dissemination. Although GTNs commonly follow a molar pregnancy, they can occur after any gestational event, including induced or spontaneous abortion, ectopic pregnancy, or term pregnancy. The study was conducted to know the incidence of different types of gestational trophoblastic diseases in the local population and the percentage of people ultimately requiring chemotherapy.

Methods: The retrospective analysis of case record of 124 women with a diagnosis of GTD admitted to Karnataka Institute of Medical Sciences Hubli between November 2008 to November 2017.

Results: A total of 124 cases of GTD were reviewed. Hydatidiform mole was diagnosed in 91 patients; of those experienced spontaneous remission after evacuation. 04 patients had persistent gestational trophoblastic Neoplasia and 13 cases of invasive mole (GTN) 1 case of epitheloid trophoblastic tumors and 15 cases of choriocarcinoma 99 (80\%) had low-risk GTN, 25 (20\%) had high-risk GTN.

Conclusions: Hydatidiform mole was found to be the most common form of gestational trophoblastic diseases. Majority of the cases got cured by simple surgical evacuation. During the course of our study some rare cases of gestational trophoblastic diseases were noted. Patients' compliance for serial follow up is a highly challenging task in developing countries. Registration of women with GTD represents a minimum standard of care.
\end{abstract}

Keywords: Gestational trophoblastic disease, Hydatidiform mole, Invasive mole

\section{INTRODUCTION}

The gestational trophoblastic diseases encompass a wide range of conditions that vary in their clinical presentation, their propensity for spontaneous resolution, local invasion and metastasis and their overall prognosis. Gestational trophoblastic diseases are the lesions of trophoblasts with varying proliferative capacities ranging from Non-neoplastic hydatidiform mole (complete hydatidiform mole, partial hydatidiform mole, invasive mole) to bonafide neoplastic conditions gestational choriocarcinoma, placental site trophoblastic tumour and Epithelioid trophoblastic tumour., ${ }^{1,2}$ In addition, two tumour like conditions are also included i.e exaggerated placental site reaction and placental site nodule or plaque. $^{3}$ The highest incidence of hydatidiform mole per 1000 pregnancies is seen in South East Asia with rates ranging from 13 in Indonesia, 8 in Taiwan, 5 in Philippines and 3.8 in Japan. ${ }^{4-8}$ North America. ${ }^{9-11}$ Europe, and Oceania have the lowest incidence with approximately $0.5-1.84 / 1000$ pregnancies. ${ }^{12,13}$ The incidence of choriocarcinoma varies from 1 in 40,000 
pregnancies in North America to 3.2/40,000pregnancies in South East Asia and Japan ${ }^{3}$

The aim of this study was to review all molar pregnancies admitted at our institution and to study the incidence, clinical presentation, management, complications and outcome of molar pregnancies.

\section{METHODS}

The Retrospective study was conducted at a tertiary care centre Karnataka Institute of Medical Sciences, Hubli, Karnataka in the Department of Obstetrics and Gynaecology. Total of 124 cases which were histologicaly diagnosed to have gestational trophoblastic diseases from November 2008 to November 2017 were included in the study. Data was collected from their respective medical records and analysed with simple percentage method.

The clinical records of patients with molar gestation were analysed regarding age, parity, gestational age at presentation, history, mode of presentation, investigations, management and follow up. Pre-treatment evaluation was done with complete haemogram, blood grouping and typing, coagulation profile, liver function test, renal function test, thyroid function test, baseline serum Hcg levels and chest X ray. CT/MRI was not routinely done unless there was evidence of extra uterine disease. Confirmation of diagnosis was made by pelvic ultrasound quantitative serum beta Hcg testing and histological examination of products of conception. WHO scoring was done and treated accordingly. Patients diagnosed at our hospital and receiving treatment outside were excluded from the study.

\section{RESULTS}

In this study total number of deliveries was $10,08,576$ from November 2008 to November 2017. Total of 124 patients were diagnosed with molar pregnancies. The incidence was 1.2/1000 pregnancies.

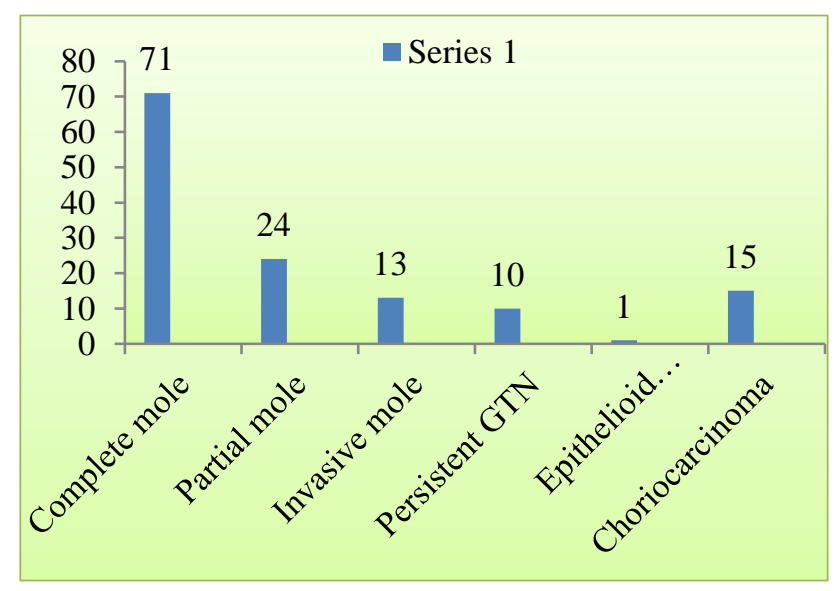

Figure 1: Distribution of cases of GTN.
Out of 124, 95 patients were diagnosed as hydatidiform mole, out of which 10 Patients had persistent GTN, 13 cases were diagnosed as invasive mole, 15 cases of choriocarcinoma and 1 case of epitheloid trophoblastic tumour was diagnosed.

Maternal age is perhaps the most important for development of molar pregnancy. In our study nearly $50 \%$ of cases were constituted by extremes of age. Mean age of presentation was 23 years. Oldest women with GTD were 45 years old. Majority were nulliparous patients (Figure 3). Vaginal bleeding was the most common symptom (Figure 4). Theca lutein cyst was present in 27.41 percentage of patients.

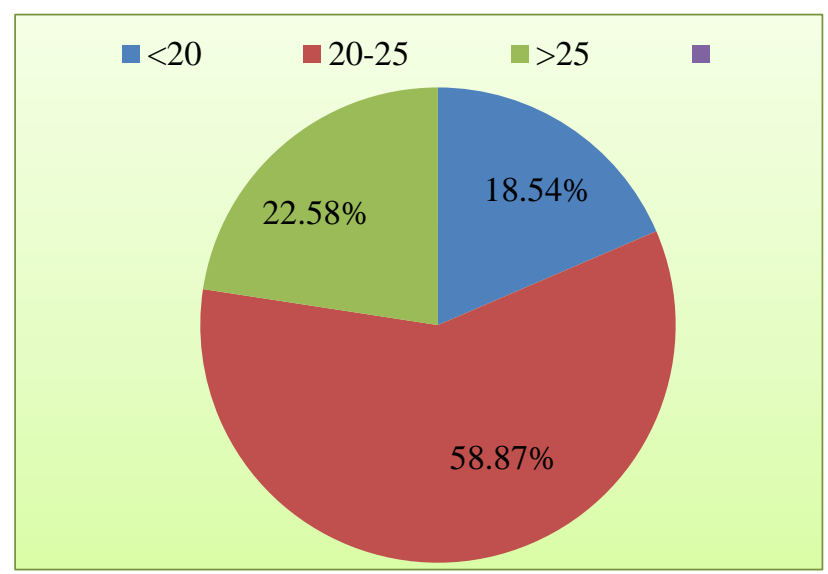

Figure 2: Distribution by age.

Gravidity, parity, smoking exposure to herbicides, paternal age, viral infections, blood types are not significantly associated with the development of Hydatidiform mole. ${ }^{6} 52.8 \%$ patients did not have prior pregnancy, while $25.7 \%$ patients had full term deliveries and $19.5 \%$ patients had prior abortion.

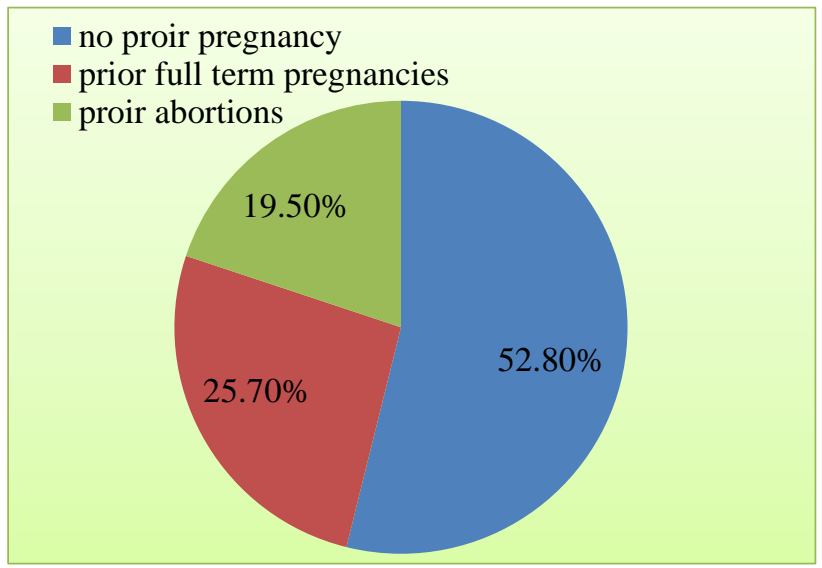

Figure 3: Antecedent pregnancy.

GTD causes a wide spectrum of different symptoms. Majority of Hydatidiform moles that undergo ultrasound examination are not recognised as molar on scan and are diagnosed as missed miscarriage or early pregnancy 
failure clinically and sonographically and the initial diagnosis of molar disease being made by the pathologist reporting the routine histopathological assessment of products of conception from the failed pregnancies.

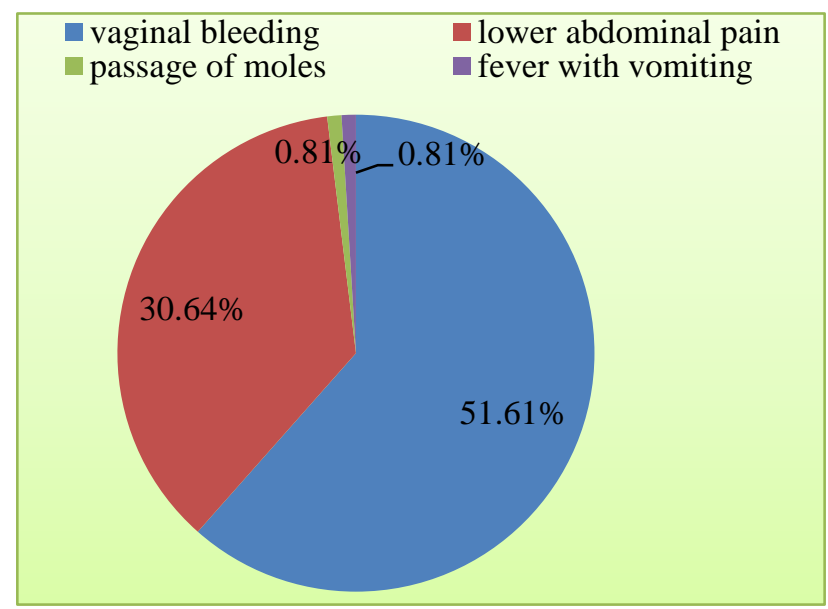

Figure 4: Symptoms at presentation.

With the routine early pregnancy ultrasound scans and ultrasound being used as the first line investigation for women presenting with vaginal bleeding, it is now uncommon for Hydatidiform mole to present with features such as enlarged uterus and other changes secondary to advanced disease. The most frequent presentation is either nonspecific bleeding followed by diagnosis of failed pregnancy or incidental detection of miscarriage on ultrasound examination. In THIS study $48.38 \%$ patients had uterine size larger than dates.

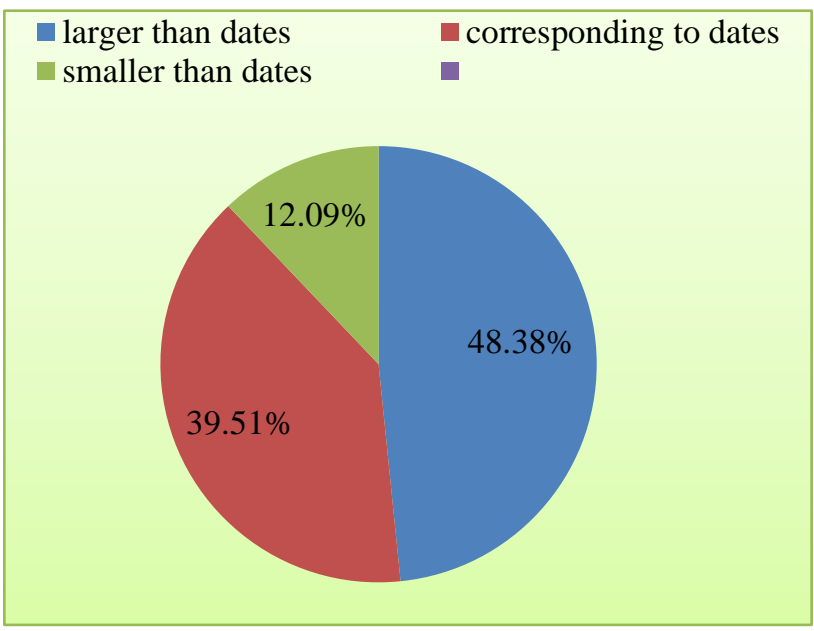

Figure 5: Uterine size with gestational age.

Baseline beta $\mathrm{Hcg}$ values at admission was recorded. $51.61 \%$ patients had beta Hcg value >2, 00,000. Serum TSH level was high in $25 \%$ of patients.

According to FIGO Staging, $80 \%$ of patients had scores less than 7 and were categorised as low risk.20\% of patients had scores 7 to 13

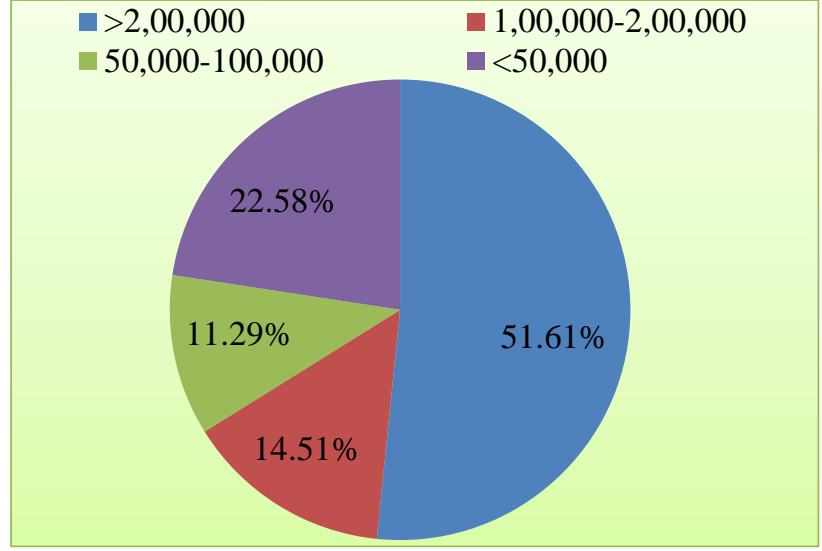

Figure 6: Serum beta HCG levels.

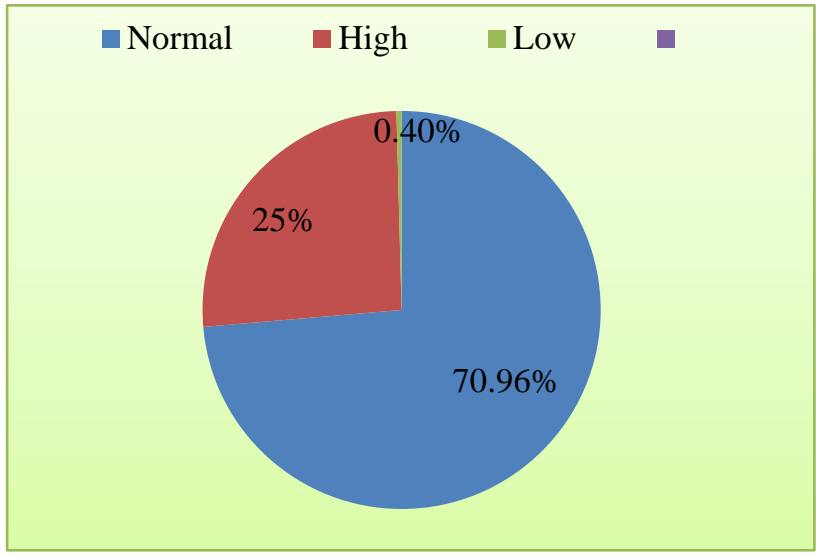

Figure 7: Serum TSH levels of patients with molar gestation.

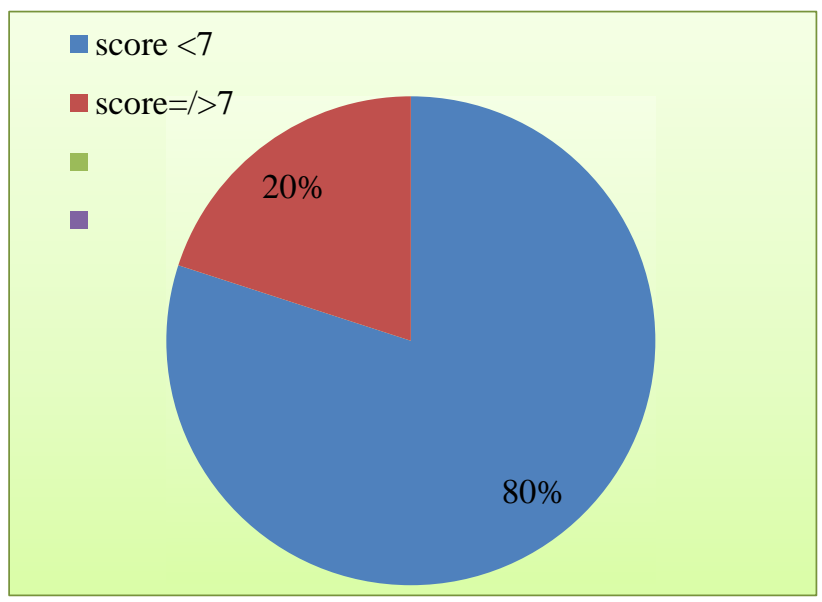

Figure 8: Percentage of high risk patients.

Majority of the patients (68.54\%) with Hydatidiform mole were cured with suction and curettage with normalisation of beta-hcg values. Ten patients with complete mole with persistently elevated hCG values were treated with single agent chemotherapy Methotrexate with leucovorin with subsequent normalization of beta-Hcg values. In the low-risk group the first-line treatment was Methotrexate with leucovorin, 
with a complete response rate of $100 \%$. Methotrexate chemotherapy was given for 10 cases of persistent GTN, 13 cases of invasive mole, 1 case of epithelioid trophoblastic tumour and 5 cases of choriocarcinoma. High-risk patients who's WHO prognostic scores were 713 were treated initially with EMA/CO and few cases underwent TAH (Table 1).

Table 1: Indications for hysterectomy.

\begin{tabular}{|ll|}
\hline Cause & Number of cases \\
\hline Perforation & 03 \\
\hline Heavy bleeding & 03 \\
\hline Epitheloid tumour & 01 \\
\hline Mass per abdomen (20 weeks) & 01 \\
\hline
\end{tabular}

Compliance was near $98.1 \%$. One woman died of CNS metastasis. Other women died on post-operative day 2 due to massive internal bleeding with DIC due to perforating mole.

\section{Management of gestational trophoblastic diseases}

\section{Hydatidiform mole}

- Eighty-five patients responded with suction and evacuation.

- Ten patients with persistently elevated beta hcg levels responded to methotrexate chemotherapy.

- Three patients underwent hysterectomy due to heavy bleeding during suction evacuation.

Invasive mole

- Thirteen patients were treated with Methotrexate chemotherapy.

- Three patients underwent hysterectomy.

\section{Choriocarcinoma}

- Ten cases responded with EMACO regimen.

- Four cases responded with Methotrexate chemotherapy.

- Hysterectomy was done in 1 patient followed by methotrexate chemotherapy.

Patient with history evacuation for molar pregnancy presented with irregular $\mathrm{p} / \mathrm{v}$ bleeding on and off. After admission perforation with massive hemoperitoneum was detected and emergency laparotomy with hysterectomy was done. Intraoperatively there was invasion into the whole of uterus. She was given massive transfusion and she recovered with normalisation of beta Hcg levels.

A 45 years old patient was admitted with history of abdominal pain and retention of urine. Diagnosis at the time of admission was adenomyosis or fibroid uterus with degenerative changes. Total Abdominal Hysterectomy was done. Intraopertatively uterus 20 weeks size with significant bulge on posterior wall with serosal thinning and blackening (Figure 2). Vertical incision was taken over uterus. There was no cleavage between fibroid and capsule. Histopathology of Uterine mass shows a tumor composed of trimorphic malignant trophoblastic cells consisting trophoblast of intermediate trophoblast, cytotrophoblast and syncytiotrophoblast with marked hemorrhage and necrosis. Brisk mitotic activity, bizarre cells, lymph vascular invasion, tumor infiltrating up to serosa. Both anterior and posterior lips were free from tumor. Patient responded with postoperative Methotrexate chemotherapy.

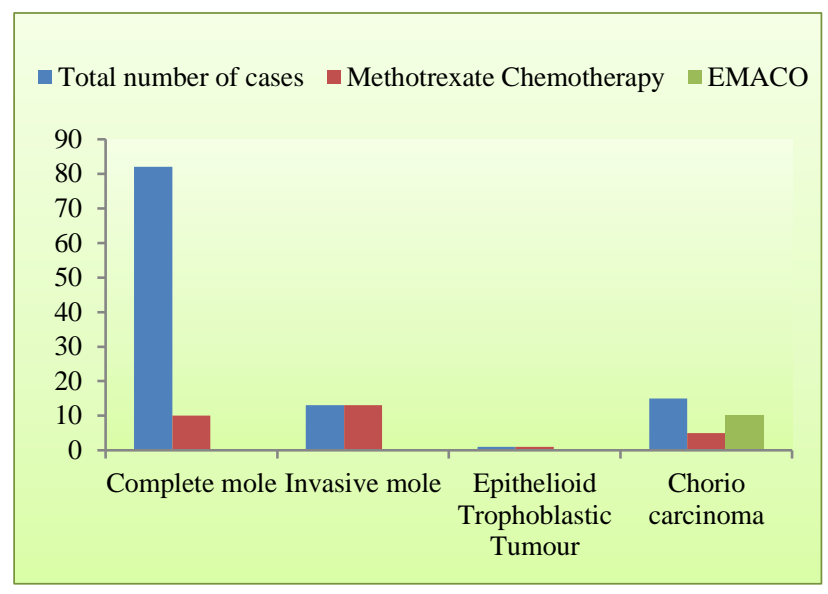

Figure 9: Number of patients who required chemotherapy.

\section{DISCUSSION}

All forms of gestational trophoblastic diseases including molar pregnancies are rare and their aetiology, biology, responsiveness to treatment are very different from other forms of malignancy. At present there is no effective prognostic method that allows accurate distinction between the patients who will develop invasive disease after evacuation and the majority who will not. Histological diagnosis of hydatidiform mole particularly partial mole is far from being accurate compounded by high risk percentage of so called genetic partial mole that are clinically unrecognised as missed abortion. On contrary significant percentage of gestations are over diagnosed as partial mole. Because of such diagnostic inaccuracy epidemiology of GTD cannot be accurately assessed with currently available data. ${ }^{3}$ Most recent development of cost effective DNA genotyping has drawn greater attention as a highly practical and accurate method of diagnosis particularly sub typing of hydatidiform mole that will overcome current diagnostic problems. ${ }^{3}$ As a result all patients with molar pregnancy should be registered for an HCG follow up system.

According to FIGO, women with partial hydatidiform mole should be followed up biweekly beta Hcg controls to record a normal level of beta-Hcg in two consecutive measurements followed by monthly check up for 3-6 months. Complete hydatidiform mole requires monthly 
checkups for an entire year ${ }^{14}$. Patients should be instructed to use contraception for a period of at least one year to enable conclusive follow-up process. Intra uterine devices should be avoided for risk of uterine perforation. $^{14}$ Usage of hormones during pending elevated beta-Hcg values can be recommended. ${ }^{14}$

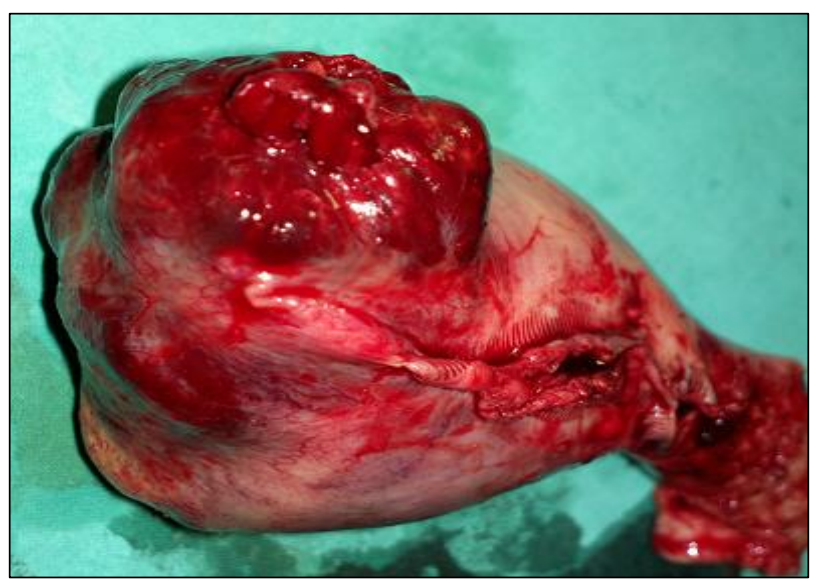

Figure 10: Epetheliod trophoblastic tumour.

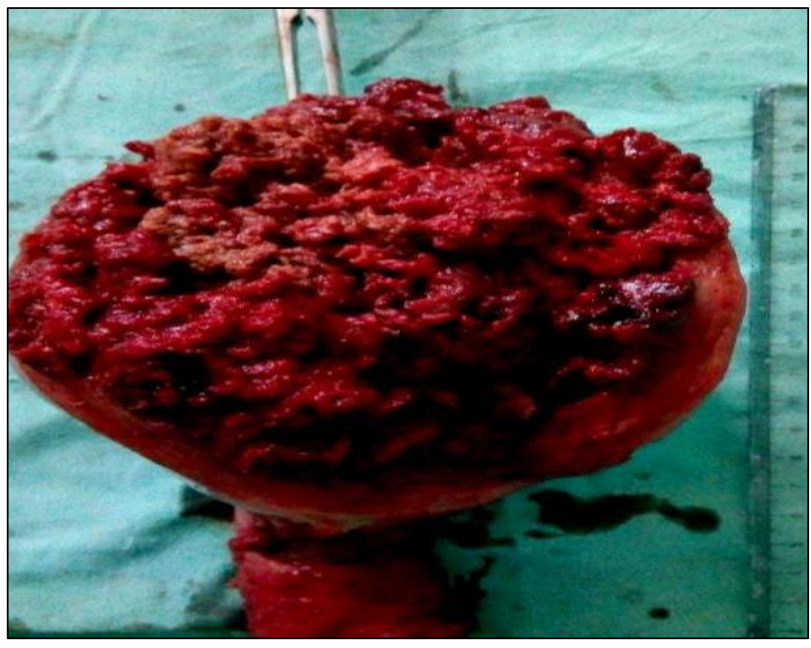

Figure 11: Choriocarcinoma uterus (non-gestational).

Thorough evaluation and staging allow selection of nonmetastatic (Stage I) and low risk metastatic (stages II, III, score <7) GTD can be treated with single agent chemotherapy resulting in survival rate approaching $100 \%$. High risk GTN (Stage II-IV Score=7/>7) require initial multi agent chemotherapy with or without adjuvant radiation or surgery to achieve a survival rate of 80 90\%. ${ }^{15}$ Approximately $30 \%$ of high risk patients will fail first line chemotherapy or relapse from remission. ${ }^{15}$ Most of these patients will have a clinic pathological diagnosis of choriocarcinoma, multiple metastases to sites other than lungs, vagina or inadequate chemotherapy. Salvage chemotherapy with drug regimens combining platinum agents Etoposide, Bleomycin, Ifosfamide or paclitaxel often in conjunction with surgical resection of sites of persistent tumour will result in cure of most of these high risk patients. ${ }^{15}$ Newer anticancer drugs such as Paclitaxel,
Gemcitabine or high dose chemotherapy may have a role in the future management of selected patients. ${ }^{15}$ Gestational trophoblastic Neoplasia occurs in $10 \%$ of women with molar pregnancy. ${ }^{16}$ Patients' compliance for serial follow up is a highly challenging task in developing countries. All women diagnosed with GTD should be provided with written information about the condition and the need for referral for follow up to a trophoblastic screening centre should be explained. ${ }^{17}$ Registration of women with GTD represents a minimum standard of care. ${ }^{17}$ After registration, follow up consists of serial estimation of HCG levels, either in blood or urine specimens. $^{17}$

\section{CONCLUSION}

Hydatidiform mole was found to be the most common form of GTD. Appropriate diagnosis and treatment leads to near $100 \%$ cure. Monitoring with serial beta-Hcg values forms the cornerstone in the management of Gestational trophoblastic diseases. A national LEVEL registry should be maintained to monitor and follow up the cases. During the course of our study some rare cases of gestational trophoblastic diseases was noted which included choriocarcinoma with brain metastasis without lung involvement, Epitheloid trophoblastic tumour Choriocarcinoma uterus (Non-gestational) Invasive mole and invasive mole with silent uterine perforation, familial recurrent hydatidiform mole (recurred thrice in same patient). Persistent GTN with multiple pseudo aneurysm over the abdomen. Choriocarcinoma deposits in posterior vaginal wall without any obvious primary, tubal ectopic molar pregnancy and placental site nodular reaction.

GTN is a curable disease. The quality of care following international guidelines and multidisciplinary dedicated teams are cornerstones to achieve successful results.

\section{Funding: No funding sources \\ Conflict of interest: None declared \\ Ethical approval: Not required}

\section{REFERENCES}

1. Kaji T. Ohama K. Androgenetic origin of Hydatidiform mole. 1997:268:633-4.

2. Czernobilsky B. Barash A, Lancet M. Partial moles a clinicopathological study of 25 cases. Obstet Gynecol. 1982:59:75-7.

3. Hui P. Gestational Trophoblastic Disease Diagnostic and Molecular Genetic Pathology, Dec 2011,1-179.

4. Sebire NJ, Fisher RA, Rees HC. Histopathological diagnosis of partial and complete hydatidiform mole in the first trimester of pregnancy. Pediat Develop Pathol. 2003 Jan 1;6(1):69-77.

5. Seckl MJ, Dhillon T, Dancey G, Foskett M, Paradinas FJ, Rees HC, et al. Increased gestational age at evacuation of a complete hydatidiform mole: does it correlate with increased risk of requiring chemotherapy?. J Reprod Med. 2004;49(7):527-30. 
6. Khoo SK Siddhu M, Bartz D. Persistence and malignant squeal of gestational Trophoblastic disease, clinical presentation, diagnosis, treatment and outcome. Aust NZJ Obst Gynecol. 2010:50:816.

7. Newland SES, Holden L, Seckl M, Strickland S, Rustin G. Management of brain metastasis in patients with high risk gestational trophoblastic diseases Reprod Med. 2002;47:465-71

8. Ngan S, Seckl MJ. Gestational trophoblastic neoplasia management: an update. Current opinion in oncology. 2007 Sep 1;19(5):486-91.

9. Sebire NJ, Lindsay I. Current issues in histopathology of gestational trophoblastic tumour. 2010:29:30-4

10. Leisorowitz GS, Webb MJ. Treatment of PSTT with hysterotomy, uterine Reconstruction Obstet Gynecol Oncol. 1996;8:696-9.

11. Tsuji Y, Tsubamohn H, Ogasawara T, Koyama K. Case of PSTT treated with chemotherapy followed by open uterine tumour resection to preserve ferility. Gynecol Oncol. 2002;87:303-7.

12. van Trommel NE, Massuger LF, Verheijen RH, Sweep FC, Thomas CM. The curative effect of a second curettage in persistent trophoblastic disease: a retrospective cohort survey. Gynecologic oncology. 2005 Oct 1;99(1):6-13.
13. Alazzam MI, Tidy J, Hancock BW, Osborne R. First line chemotherapy in low risk gestational trophoblastic neoplasia. Cochrane Database Syst Rev. 2009 Jan 1;1.

14. Stevens FT, Katzorke N, Tempfer C, Kreimer U, Bizjak GI, Fleisch MC, et al. Gestational trophoblastic disorders: an update in 2015. Geburtshilfe und Frauenheilkunde. 2015 Oct;75(10):1043.

15. Lurain JR. Gestational Trophoblastic Disease II Classification, Management of Gestational Trophoblastic Neoplasia. AMJ Obst Gynecol. 2011;204(1):11-8.

16. Sebire NJ, Seckl MJ. Gestational trophoblastic disease: current management of hydatidiform mole. Bmj. 2008 Aug 15;337:a1193.

17. Tidy J, Hancock BW. The management of gestational trophoblastic disease. Green-Top Guideline. 2010(38):1-1.

Cite this article as: Antaratani RA, Shruthi M. Study of gestational trophoblastic diseases at a tertiary care hospital in India. Int J Reprod Contracept Obstet Gynecol 2018;7:1622-7. 